\title{
Streamlined Q-M Method
}

This paper was downloaded from TechRxiv (https://www.techrxiv.org).

\section{LICENSE}

CC BY 4.0

SUBMISSION DATE / POSTED DATE

02-01-2022 / 06-01-2022

CITATION

olivier, philip (2022): Streamlined Q-M Method. TechRxiv. Preprint.

https://doi.org/10.36227/techrxiv.17712188.v1

$\mathrm{DOI}$

10.36227/techrxiv.17712188.v1 


\title{
Streamlined Q-M Method \\ Philip D Olivier \\ philip_d_olivier@ieee.org
}

\begin{abstract}
This paper presents a streamlined version of the Quine-McCluskey tabular process for simplifying a Boolean expression. The simplification comes from: a) avoiding grouping processes; b) using a purely numeric notation for simplified implicants; $c$ ) the numeric notation allows the "covered" min terms to be generated at the end of the process thereby eliminating the need to track which simplified implicants "cover" which min terms. Examples are presented.
\end{abstract}

\section{Introduction}

A canonical sum-of-products representation of a logic function (see [2]) summarizes the truth table of the function. It requires a large number of AND, OR and NOT gates to implement in hardware. Methods have been developed to reduce the number of AND, OR and NOT operations required and hence to reduce the cost of implementation. One such method, the Quine-McCluskey tabular method, can be summarized as

1. Given the min term implicants of a Boolean function $f=\sum m(i, j, \ldots)$

2. Find the prime implicants of $f$

3. Find the minimal covering of $f$ using the prime implicants of $f$

These steps are accomplished using tables that

a. Make it easy to see how to construct the prime implicants

b. Make it easy to see which prime implicants cover the min term implicants.

The purpose of this paper is to revisit the problem of simplifying a Boolean algebra expression initially given in Sum-of-Products (SoP) form into a different, simpler, SoP form. The desired solution should be implementable in software (MatLab in this paper) using only integer andBoolean arithmetic; it should avoid non numeric symbols except during the "pretty print" stage.

The term simpler is quantified by counting the number of basic logical operations of AND, OR, and NOT. Each of these logical operations can be mechanized by corresponding electronic components, or gates, with the corresponding name. The fewer the gates, the simpler the result.

It is not the purpose of this paper to translate the Q-M tabular method into computer code. It is the purpose of this paper to produce computer code that accomplishes steps 1, 2, and 3 above in a different way.

The reader can then decide if it is indeed "streamlined". 
The reader is assumed to be familiar with digital logic at the level typically taught in $2^{\text {nd }}$ year electrical engineering courses (see B\&V). Two techniques for simplifying logic functions covered in such a course are the Karnaugh Map (KMap)technique and a tabular method often associated with the names Quine and McCluskey (Q-M method or process). Some of the notation used is summarized in Figure 1.

The Q-M process for finding prime implicants has several salient features

- Grouping and sorting to produce tables that make it easy to find implicants that can be combined. Implicants that are likely to be combinable are in sequential groups.

- Non-numeric coding to indicate that a combination has been formed for example $a b^{\prime} c d+a b^{\prime} c^{\prime} d=a b^{\prime} d$ gets coded as $1011+$ $1001=10-1$

This paper simplifies the Q-M process by not performing the grouping and sorting that produce the tables it is famous for. The grouping and sorting is replaced by exhaustive comparisons. Computers are very good at exhaustive comparisons, humans are not.

The binary representation of the min terms and implicants is accomplished using row vectors, the non-numeric coding is replaced by using the numeric " 10 " in place of the "- " in the location of the missing literal in the vector representing the combined implicant.

\section{Some Definitions}

These definitions can be found in any basic book on Digital Logic, see [2] for example.

Canonical Sum-of-Products. Consider a logic function of the three variables $a, b$, and c, defined by a full truth table. Each row of the truth table contains the explicit values of the variables e.g. 010 , and the associated truth value of
Min terms, Boolean values, Logical expressions

\begin{tabular}{|c|c|c|c|c|}
\hline \multirow{2}{*}{$\begin{array}{l}\min \\
\text { term }\end{array}$} & \multicolumn{3}{|c|}{ variable values } & \multirow{2}{*}{$\begin{array}{c}\text { logical } \\
\text { expression }\end{array}$} \\
\hline & a & $b$ & C & \\
\hline $\mathrm{m}_{0}$ & 0 & 0 & 0 & $a^{\prime} b^{\prime} c^{\prime}$ \\
\hline $\mathrm{m}_{1}$ & 0 & 0 & 1 & $a^{\prime} b^{\prime} c^{\prime}$ \\
\hline $\mathrm{m}_{2}$ & 0 & 1 & 0 & $a^{\prime} b c^{\prime}$ \\
\hline$m_{3}$ & 0 & 1 & 1 & $a^{\prime} b c$ \\
\hline $\mathrm{m}_{4}$ & 1 & 0 & 0 & $a b^{\prime} c^{\prime}$ \\
\hline$m_{5}$ & 1 & 0 & 1 & $a b^{\prime} c^{\prime}$ \\
\hline $\mathrm{m}_{6}$ & 1 & 1 & 0 & $a b c^{\prime}$ \\
\hline $\mathrm{m}_{7}$ & 1 & 1 & 1 & $a b c$ \\
\hline
\end{tabular}

Sample Truth Table

\begin{tabular}{|c|c|c|c|}
\hline$a$ & $b$ & $c$ & $f$ \\
\hline 0 & 0 & 0 & 1 \\
\hline 0 & 0 & 1 & 0 \\
\hline 0 & 1 & 0 & 0 \\
\hline 0 & 1 & 1 & 1 \\
\hline 1 & 0 & 0 & 1 \\
\hline 1 & 0 & 1 & 0 \\
\hline 1 & 1 & 0 & 0 \\
\hline 1 & 1 & 1 & 0 \\
\hline
\end{tabular}

Each row is an IF-THEN statement.

$1^{\text {st }}$ row: If $a=0$ and $b=0$ and $c=0$, then $f=1$. $6^{\text {th }}$ row: if $a=1$ and $b=0$ and $c=1$, then $f=0$. The values of $a, b, c$, imply the value of $f$.

$$
\begin{gathered}
f=1 m_{0}+0 m_{1}+0 m_{2}+1 m_{3}+1 m_{4} \\
+0 m_{5}+0 m_{6}+0 m_{7} \\
f=\sum m(0,3,4)=a^{\prime} b^{\prime} c^{\prime}+a^{\prime} b c+a b^{\prime} c^{\prime} \\
f=b^{\prime} c^{\prime}+a^{\prime} b c
\end{gathered}
$$

An implicant is set of variable values that imply $f=1$.

The min term implicants are $m_{0}, m_{3}, m_{4}$

Figure 1. Notation summary. 
the function, whether it is 0 or 1 (perhaps ' $d$ ' for do not care). The string of 0 's and 1's that describe input values can be viewed as a binary number so one can construct the minterm associated with that row

$$
010 \leftrightarrow 010_{2} \leftrightarrow 2_{10} \leftrightarrow a^{\prime} b c^{\prime} \leftrightarrow m_{2} .
$$

With this notation, the canonical sum-of-products form for the function $f$ can be expressed as

$$
f(a, b, c)=\sum_{i=0}^{2^{3}-1} f_{i} m_{i}
$$

Where

- $f_{i}=0,1$, or $d$, for FALSE, TRUE, or do-not-care respectively. See Figure 1.

An implicant is a Boolean expression that guarantees that the function is true, i.e. it implies that the function is true. Implicants can be combined to produce other implicants. E.g., if the three variable expressions a'bc and a'b'c are implicants, they can be combined to produce a'c which is an implicant and it has only 2 variables. Separately, the expression a'bc $+a^{\prime} b^{\prime} c$ requires 4 AND gates, 1 OR gate and 2 NOT gates. After combination, the expression a'c requires 1 AND gate and 1 NOT gate.

A Prime Implicant Is an implicant that cannot be combined with any other implicant to produce an implicant with fewer literals (variables whether negated or not).

An Essential Prime Implicant is a prime implicant that MUST be included in any simplification of the function.

Problem: Given a canonical SoP representation of a Boolean function, $f(a, b, c, \ldots)$. Find a reduced/simplified SoP representation assuming a computer-based implementation. I.e. convert (each $m$ or $d$ has the form a'bcd which requires 3 ANDs)

$$
f(a, b, c, \ldots)=\sum m(j, \ldots)+\sum d(k, \ldots)
$$

into something like

$$
f(a, b, c, \ldots)=a c+d^{\prime} f .
$$

The Boolean form of a min term implicant is the most basic of implicants. That is, when it is TRUE it implies that the function is true. Further it specifies only one way the function is TRUE.

Consider the Boolean AND expressions in the variables $a, b, c, \ldots$ where

$$
a^{\prime}=\operatorname{not}(a), \quad a b=a \text { AND } b, \quad a+b=a \text { OR } b .
$$


The proposed algorithm includes consideration of do-not-cares. The "+" symbol is overloaded, that is, it is used in two distinct ways: it can mean logical OR; additionally, it can also mean traditional integer addition.

\section{Boolean Algebra Basis}

Two particular properties unique to Boolean Algebra (BA) are used repeatedly in the proposed process. These are the same BA properties that undergird the simplification processes associated with Karnaugh Maps (K Maps) and the Quine-McCloskey (QM) tables.

1) A term can be re-used as often as desired/useful.

2) ORing a term with its negation produces a Boolean 1.

$A=A+A=A+A+A=\cdots$

$A x+A x^{\prime}=A\left(x+x^{\prime}\right)=A(1)=A$

Distributivity, associativity, commutivity, and the fact that 1 is the multiplicative identity are also used, but these are holdovers from real valued algebra familiar from High School.

The Streamlined Q-M method, (SQM for short) is presented here. It is simplified in several ways

1. No sorting into groups or Gray coding of the inputs is required

2. It is based on representing implicants as row vectors.

3. The same representation is used for min term implicants as for prime implicants and for intermediate implicants.

4. A function is provided that can compute the min term implicants that are covered by a Prime implicant.

5. The prime implicants that cover the least covered min term implicants are chosen to be included in the simplified function.

6. No symbolic processing is used. Only integer and Boolean operations are used.

7. Only at the very end, i.e. at the "pretty print" stage are non-numeric symbols used.

\section{Numerical representation of Boolean Products}

Notation and how the "real" problem is encoded in numerical structures play an important part in any algorithm implemented in software. In particular, this algorithm needs to be able to encode Boolean products such as $a b^{\prime} c d$ and $a b d$ in a simple, easy to manipulate way. A numeric row vector representation is chosen. Each Boolean AND expression is assumed to have its variables in a particular, unchanging order. (Typically, alphabetic order or increasing numerical order by subscripts is used.) Each slot in the row vector holds one of three values, depending on how the corresponding variable appears in the AND expression. There are three possibilities:

1) If the variable appears as itself, then a 1 appears in its slot;

$\mathrm{b}<->1$;

2) If the variable appears negated, then a 0 appears in its slot;

$b^{\prime}<->0$;

3) If the variable does not explicitly appear, then a 10 appears in its slot;

DNA $<->10$; 
Assume there are four variables (i.e. literals $a, b, c$, and $d$ or $x_{0}, x_{1}, x_{2}$, and $x_{3}$ ), each Boolean AND expression can be represented as a row vector with 4 entries as follows

$$
\text { abcd }<->\left[\begin{array}{llll}
1 & 1 & 1 & 1
\end{array}\right] \quad \text { and } \quad a b^{\prime} c d<->\left[\begin{array}{llll}
1 & 0 & 1 & 1
\end{array}\right]
$$

In case a variable does not appear in an AND expression, a "10" appears in its location in the row vector representation. The variable " $b$ " does not appear in, acd' so a 10 is put in the $2^{\text {nd }}$ slot

$$
\text { acd' }^{\prime}<->\left[\begin{array}{llll}
1 & 10 & 1 & 0
\end{array}\right]
$$

\section{Combining ORs of two Products into one Product}

The basic operation that is required is the combination of two products that differ in only one "toggled" variable. In this case, the two products can be replaced by a single product with the "toggled" variable missing. Consider the OR of two products that differ only because $d$ is "toggled", i.e. it appears as $d$ in one product and d' in the other.

$$
a b c^{\prime} d+a b c^{\prime} d^{\prime}=a b c^{\prime}\left(d+d^{\prime}\right)=a b c^{\prime}(1)=a b c^{\prime} .
$$

In the notation just introduced

$$
\left[\begin{array}{llll}
1 & 1 & 0 & 1
\end{array}\right]+\left[\begin{array}{llll}
1 & 1 & 0 & 0
\end{array}\right] \rightarrow\left[\begin{array}{llll}
1 & 1 & 0 & 10
\end{array}\right]
$$

Notice that this Boolean combination reduced the number of AND gates from 6 to 2; the number of OR gates from 1 to 0 ; and the number of NOT gates from 2 to 1 .

Observation: Two Boolean AND expressions are nicely ORable (i.e. produce a single simplified ANDing of some of their variables) provided they differ in only one location, and in that location the variable is toggled (e.g. "a" appears in one expression and "a'" in the other).

The following two pairs of $\mathrm{r} 1$ and $\mathrm{r} 2$ row vectors are representations of nicely ORable Boolean AND expressions.

abcd $<->r 1=\left[\begin{array}{llll}1 & 1 & 1 & 1\end{array}\right]$

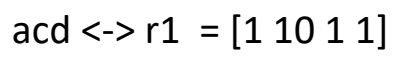

$a b c^{\prime} d<->r 2=\left[\begin{array}{llll}1 & 1 & 0 & 1\end{array}\right]$

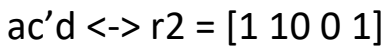

$a b\left(c+c^{\prime}\right) d<->\left[\begin{array}{llll}1 & 1 & 1 & 1\end{array}\right]$

$$
a\left(b+b^{\prime}\right)\left(c+c^{\prime}\right) d<->\left[\begin{array}{llll}
1 & 10 & 10 & 1
\end{array}\right]
$$

Observe the vector difference of these nicely ORable vectors:

$$
\begin{aligned}
& r 1-r 2=\left[\begin{array}{lll}
0 & 0 & (+1)
\end{array}\right] \\
& r 2-r 1=\left[\begin{array}{lll}
0 & 0 & (-1)
\end{array}\right]
\end{aligned}
$$

These vector differences have only one non-zero element and it is either $a+1$ or $a-1$.

Identifying ORable terms.

With this representation the signature of nicely ORable row vectors can be conveniently expressed in MatLab code as 


$$
\operatorname{sum}(\operatorname{abs}(r 1-r 2))==1
$$

Nicely ORable row vectors could be called "neighbors" a la K Maps. They would appear in sequential groups in the Q-M tabular method.

This plays a role similar to the "matching principle" used in [1].

Choosing a notation with such a nice "selection principle" as sum(abs(r1-r2)) == 1 is the key to avoiding the sorting and grouping overhead built into the Q-M tabular method.

\section{Simplified Quine McCluskey (SQM) Algorithm.}

Begin with an Implicant Matrix, IM, i.e. an array, each of its rows represents a min term in the canonical SoP representation of a Boolean Function. Also begin with a do-not-care matrix, DNC, that contains the min terms of the do-not-cares.

1 Find ALL nicely ORable rows in IM and OR them. Include the ORed results in IM1.

- Note a row can be used multiple times. (Very useful feature of Boolean algebra.)

- This is done systematically, by comparing row 1 with all succeeding rows; then comparing row 2 with all succeeding rows, etc.

- See if any row in the IM can be combined with any row in the DNC matrix. Include any such combinations in the IM1 matrix.

2 Include in IM1 the rows of IM that were not used in any of the ORs from Step 1.

- Do not include unused do-not-cares in IM1.

3 Remove repeated rows in IM1 to produce IMnew which can be used to re-start the process with IM=IMnew, DNC=[].

4 Repeat the above process until the new Implicant Matrix, IMnew, has the same total number of 10's (indicating no new ORs were possible). When complete, the rows of IMnew contain the prime implicants.

5 Note. The implicants represented by the rows of the final IM form a reduced SoP expression for the original Boolean function. From these implicants a more reduced SoP expression can normally be formed by finding the minimal covering of $f$.

6 Create a new array, Covered with as many rows as IM and as many columns as there are original min terms in $\mathrm{f}$. Each row of Covered includes the decimal subscripts of the min terms that the corresponding row of IM covers. Do not include the decimal subscripts of the DNCs it covers. Pad the end of the row with -1 's as needed.

7 Create an empty array $F$, each row of $F$ will hold a row describing a prime implicant needed to cover the original function.

8 Find a minterm that is covered by only 1 row of IM.

o Include this row in $\mathrm{F}$.

O Set that row of IM to all -1 's.

- Set the min terms covered by it to -1 .

- Repeat until all min terms are covered by 2 or more rows

9 Find row(s) of IM that cover the most uncovered min terms. Choose 1 of these rows 
Include this row in $\mathrm{F}$

Set that row of IM to all -1 's.

o Set the min terms covered by it to -1 .

- Repeat 8

○ Repeat 9

10 Repeat until all min terms are covered.

11 Pretty Print result. Assume variables are the first few letters of the English alphabet. Each row of $\mathrm{F}$ is a term separated by a Boolean "+"..

Note: any symbol other than 0 or 1 could be used to indicate that its variable does not show up. The numerical value of 10 is chosen because it is a) numeric and hence convenient in Matlab and other numeric languages and $b$ ) it mnemonically suggests both a $1<->$ a OR a $0<->a^{\prime}$. In treatments of the Quine-McClosky method a "_" or a "-" is often used, e.g. 1-10 <-> acd". Using "10" rather than "-" leads to a convenient numerical test to see if two rows can be combined.

Note: this process is analogous to the Q-M process but without pre ordering by the number of 1 's in a row. Also the ORing is accomplished by storing the numeric value of 10 in the ORed variable's location rather than using a non-numeric symbol such as a "-". The pre-ordering and non-numeric "-" used in the QM process are both very useful for human implementation. Depending on a cost-benefit analysis, the pre-ordering might be useful in the early stages of a mechanized implementation, especially for functions with many min terms.

If ordering is implemented: first order by number of 10's; then sort by those with a 10 is a particular slot; within those with the same number of 10's and a 10 in a particular slot, group by number of 1 's. There is overhead cost (additional coding as well as run time) to this ordering and grouping.

\section{Further simplification.}

Observation 1. The more 10's in a prime implicant the more min terms it can cover and the fewer AND gates it requires. During the simplification process attention needs to be focused on the least covered min terms first. Covering these will also cover other min terms, then focus on the least covered min terms, etc.

\section{Examples}

Two examples are presented. The first has more extensive comments and intermediate results. The first has 4 variables and the second 5 variables.

Example 1 from [1]. With comments. This example is taken from [1] and includes do-not-cares. Intermediate results are shown.

Problem statement: Simplify $f=\sum m(4,5,6,8,9,10,13)+\sum d(0,7,15)$ into a SoP form using prime implicants. 
Note that $f=\sum m(4,5,6,8,9,10,13)$ can be implemented with $7 * 3=21$ AND gates and 6 OR gates, and 4 NOT gates. Simpler is defined as requiring fewer gates.

Step 1. Generate the initial Implicant matrix (contains 0 's and 1's of minterms $\rightarrow f=1$ )

$$
I M 0=\left[\begin{array}{llll}
0 & 1 & 0 & 0 \\
0 & 1 & 0 & 1 \\
0 & 1 & 1 & 0 \\
1 & 0 & 0 & 0 \\
1 & 0 & 0 & 1 \\
1 & 0 & 1 & 0 \\
1 & 1 & 0 & 1
\end{array}\right], D N C=\left[\begin{array}{llll}
0 & 0 & 0 & 0 \\
0 & 1 & 1 & 1 \\
1 & 1 & 1 & 1
\end{array}\right]
$$

After combining, the prime implicants and the min terms that they cover are

$$
\text { PrimeImp }=\left[\begin{array}{cccc}
0 & 1 & 10 & 10 \\
10 & 1 & 10 & 1 \\
0 & 10 & 0 & 0 \\
1 & 0 & 0 & 10 \\
1 & 0 & 10 & 0 \\
10 & 0 & 0 & 0 \\
1 & 10 & 0 & 1
\end{array}\right] \text {, Covered }=\left[\begin{array}{ccccccc}
4 & 5 & 6 & -1 & -1 & -1 & -1 \\
5 & -1 & 13 & -1 & -1 & -1 & -1 \\
-1 & 4 & -1 & -1 & -1 & -1 & -1 \\
8 & 9 & -1 & -1 & -1 & -1 & -1 \\
8 & 10 & -1 & -1 & -1 & -1 & -1 \\
-1 & 8 & -1 & -1 & -1 & -1 & -1 \\
9 & 13 & -1 & -1 & -1 & -1 & -1
\end{array}\right]
$$

$m_{6}$ and $m_{10}$ each occur only once, so two passes through Fcover produce

$$
\begin{aligned}
& F=\left[\begin{array}{cccc}
0 & 1 & 10 & 10 \\
1 & 0 & 10 & 0
\end{array}\right] \\
& \text { PrimeImp }=\left[\begin{array}{cccc}
-1 & -1 & -1 & -1 \\
10 & 1 & 10 & 1 \\
0 & 10 & 0 & 0 \\
1 & 0 & 0 & 10 \\
-1 & -1 & -1 & -1 \\
10 & 0 & 0 & 0 \\
1 & 10 & 0 & 1
\end{array}\right] \text {, Covered }=\left[\begin{array}{ccccccc}
-1 & -1 & -1 & -1 & -1 & -1 & -1 \\
-1 & -1 & 13 & -1 & -1 & -1 & -1 \\
-1 & -1 & -1 & -1 & -1 & -1 & -1 \\
-1 & 9 & -1 & -1 & -1 & -1 & -1 \\
-1 & -1 & -1 & -1 & -1 & -1 & -1 \\
-1 & -1 & -1 & -1 & -1 & -1 & -1 \\
9 & 13 & -1 & -1 & -1 & -1 & -1
\end{array}\right]
\end{aligned}
$$

$$
\text { MTs }=\left[\begin{array}{lllllll}
-1 & -1 & -1 & -1 & 9 & -1 & 13
\end{array}\right]
$$

Now only $m_{9}$ and $m_{10}$ are uncovered. They each occur twice in the Covered matrix so we look for the prime implicant that covers the most uncovered min terms, which is the last prime implicant [1 $10 \quad 0 \quad 1]$ which produces

$$
F=\left[\begin{array}{cccc}
0 & 1 & 10 & 10 \\
1 & 0 & 10 & 0 \\
1 & 10 & 0 & 1
\end{array}\right]
$$

Converting to logic produces

$$
f(a, b, c, d)=a^{\prime} b+a b^{\prime} d^{\prime}+a c^{\prime} d
$$

A screen shot of the MatLab produced output is shown in Figure 2.

Example 2 (problem 4.7 pg 241 in [2]). Simplify $f(a, b, c, d, e)=$

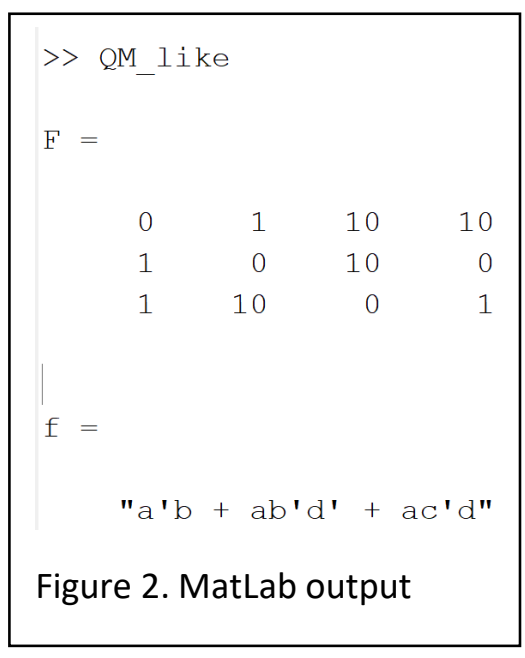
$\sum m(1,4,6,7,9,10,12,15,17,19,20,23,25,26,27,28,30,31)+\sum d(8,16,21,22)$.

The results from MatLab Code are $\mathrm{F}=$ 


$\begin{array}{ccccc}10 & 10 & 0 & 0 & 1 \\ 10 & 0 & 1 & 10 & 0 \\ 10 & 1 & 0 & 1 & 0 \\ 10 & 10 & 1 & 0 & 0 \\ 1 & 10 & 10 & 1 & 1 \\ 1 & 10 & 1 & 10 & 0 \\ 1 & 10 & 1 & 1 & 10 \\ 1 & 1 & 10 & 1 & 10\end{array}$

$f=(! c)(! d) e+(! b) c(! e)+b(! c) d(! e)+c(! d)(! e)+a d e+a c(! e)+a c d+a b d$

Note: the pretty print process was change to represent NOT(a) as (!a) rather than as a'. Note: the simplified function $\mathrm{f}$ has 8 terms. The process of combining min term implicants produced 15 prime implicants

The original canonical SoP representation requires 18 ORs, and 76 ANDs. The prime implicant SoP representation requires 14 ORs, and 34 ANDs. The SMQ reduced SoP representation requires 7 ORs, and 16 ANDs.

\section{Conclusion}

This paper presented a simplified approach to simplifying a canonical SoP representation of a logic function as compared to the traditional Quine McCluskey tabular method. The simplification follows from a new way of encoding all implicants, from min term implicants through prime implicants. This new encoding allows the process to proceed without the need for certain "overhead" processes such as grouping at each stage and keeping track of which prime implicant covers which min term implicant. The coding allows for the a simple function that can re construct the min term implicants that are covered by a given encoded prime implicant.

Comment of E-sum introduced by Vitthal and Amar in [1]. Vitthal and Amar the "e-sum" to keep track of which literal do not appear in a processed (perhaps prime) implicant. The SQM method codes this withing the representation of the implicant rather than appends the information to the representation. Their process also includes all of the grouping and tracking overhead. The work in [1] did not include a software implementation.

After running the MatLab script and seeing the

References

[1] Jadhav Vitthal and Buchade Amar, Modified Quine-McCluskey Method, arXiv pre print, 1203.2289arxiv.org. 
[2] Stephen Brown and Zvonko Vranesic, Fundamentals of Digital Logic with VHDL Design, $3^{\text {rd }}$, McGraw-Hill, 2009.

\section{Appendix: MatLab code}

\section{MatLab Code}

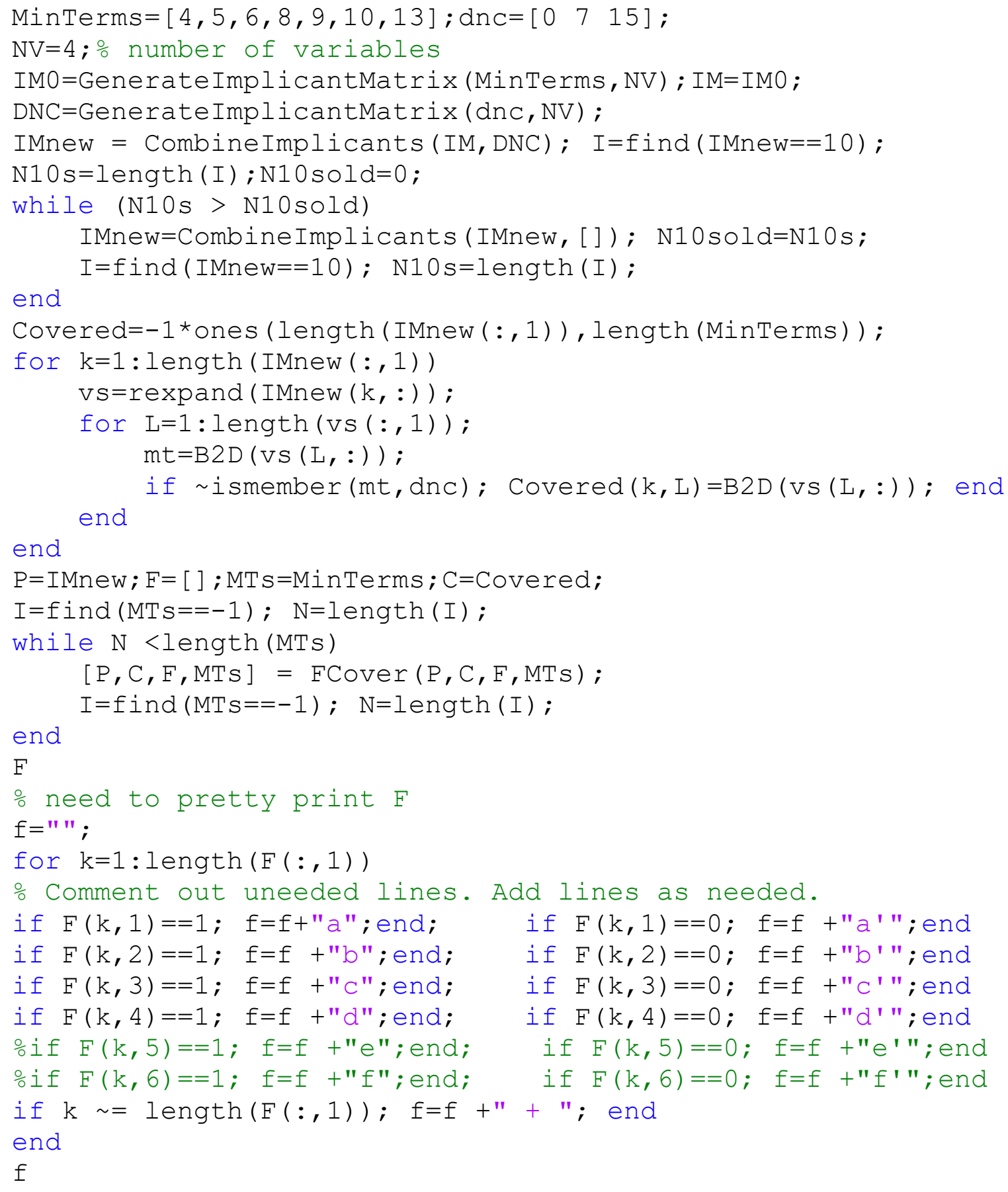




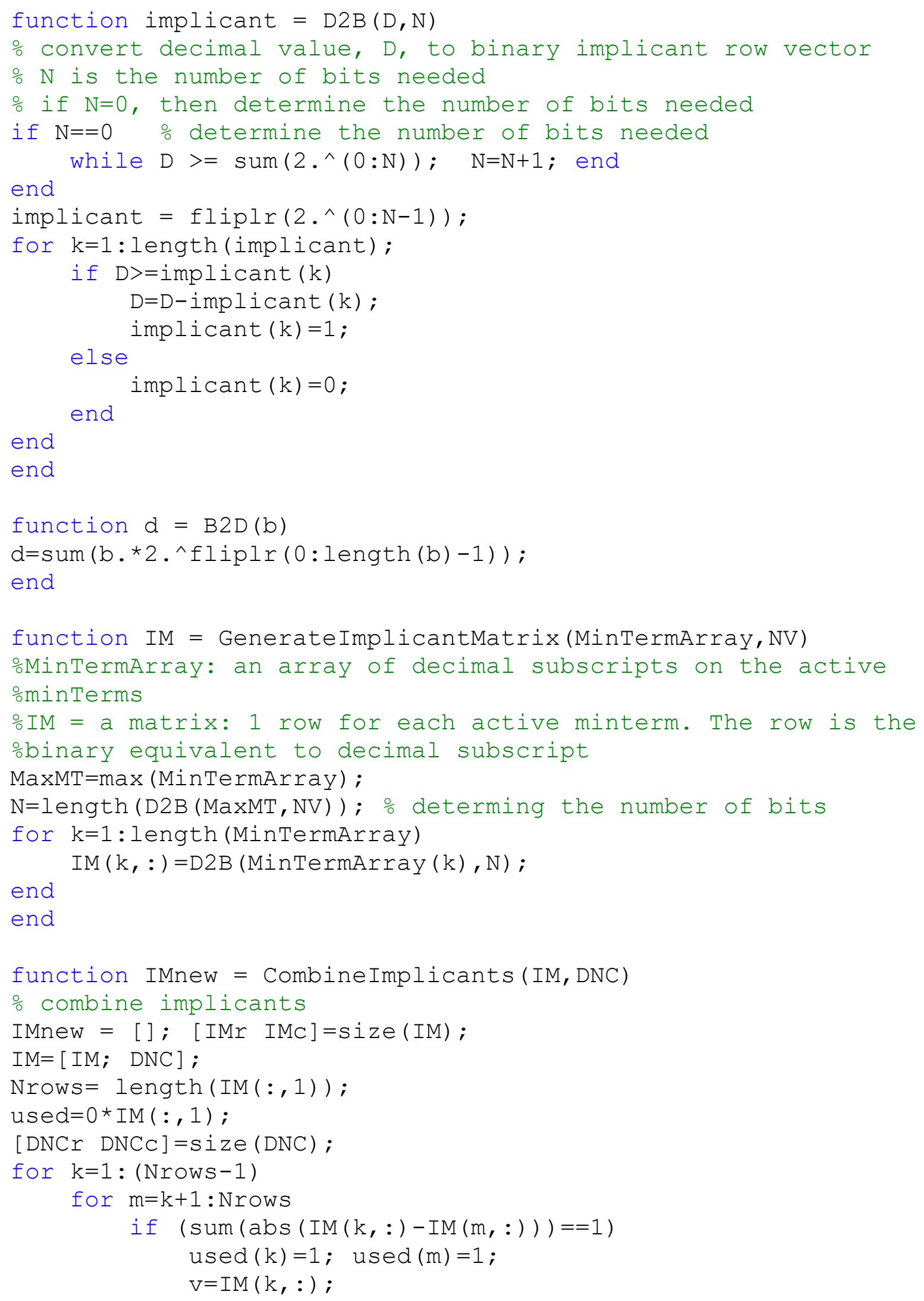




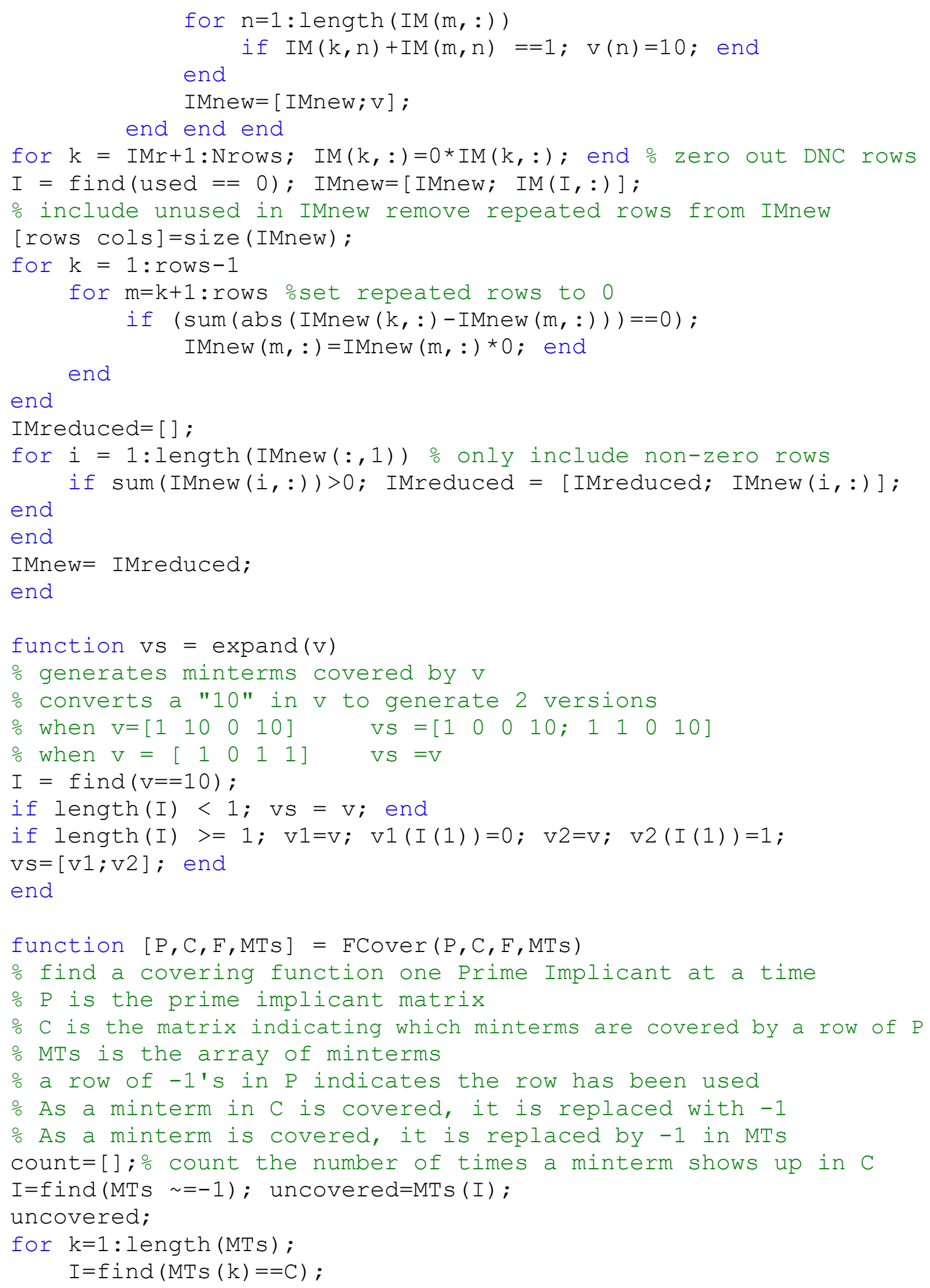




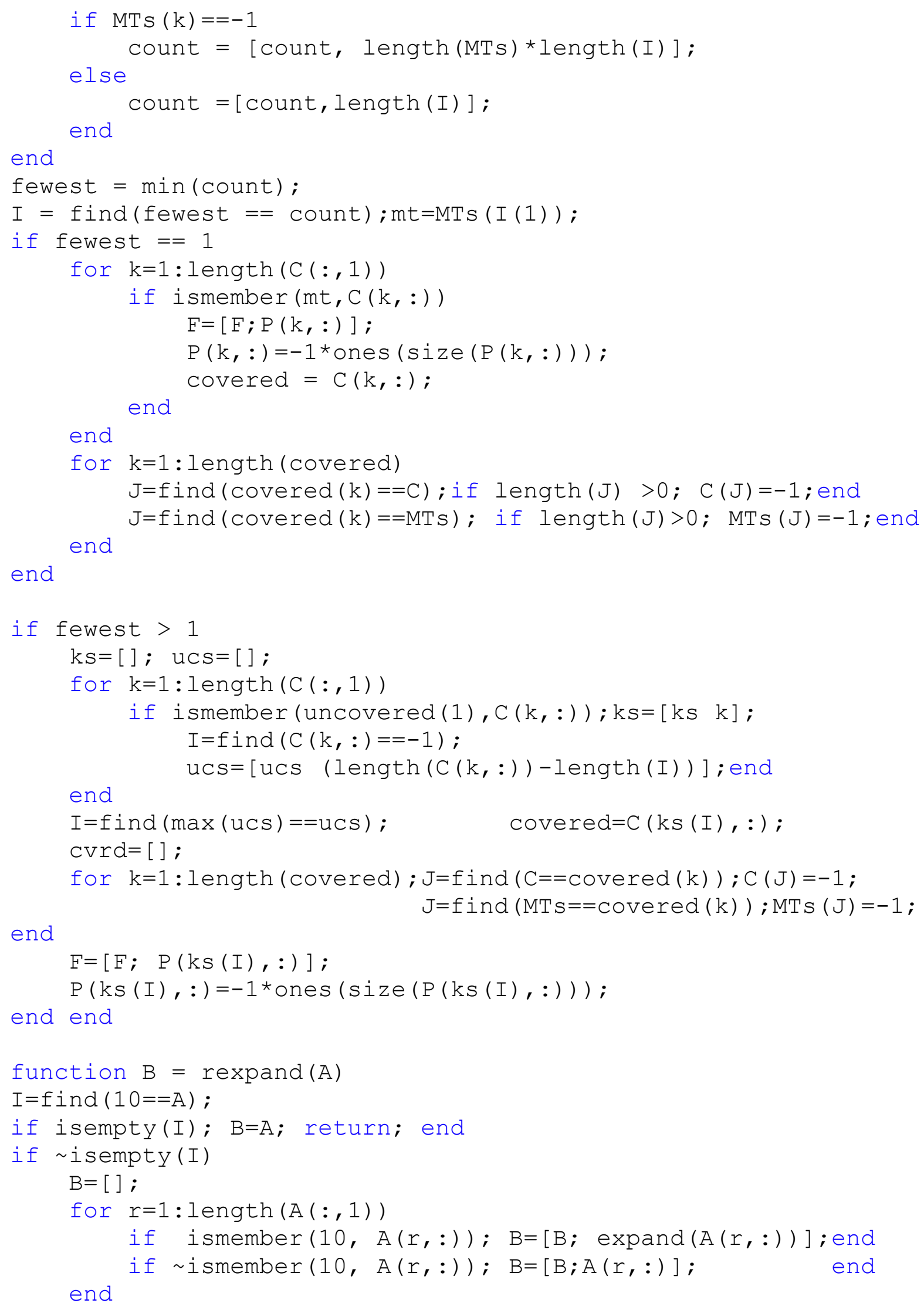

end

$\mathrm{F}=[\mathrm{F} ; \mathrm{P}(\mathrm{ks}(\mathrm{I}), \mathrm{:})]$;

$\mathrm{P}(\mathrm{ks}(I),:)=-1$ *ones $(\operatorname{size}(\mathrm{P}(\mathrm{kS}(\mathrm{I}),:)))$ );

end end 
$\mathrm{B}=$ rexpand ( $\mathrm{B})$;

end end

mo

$m_{1}$

$\mathrm{m}_{2}$

$\mathrm{m}_{3}$

$\mathrm{m}_{4}$

$\mathrm{m}_{5}$

$\mathrm{m}_{6}$

$\mathrm{m}_{7}$ 